\title{
DESENVOLVIMENTO DE METODOLOGIA ANALÍTICA PARA DETERMINAÇÃO DE COLESTEROL EM RAÇÃO PARA RUMINANTES ATRAVÉS DE PLANEJAMENTO EXPERIMENTAL FATORIAL
}

\author{
Cecília Muller Bandeira* e José Maria Ferreira \\ Departamento de Tecnologia e Inspeção de Produtos de Origem Animal, Escola de Veterinária, Universidade Federal de Minas \\ Gerais, Av. Antônio Carlos, 6627, 31270-901 Belo Horizonte - MG, Brasil \\ Neura Bragagnolo e Lilian Regina Barros Mariutti \\ Departamento de Ciência de Alimentos, Faculdade de Engenharia de Alimentos, Universidade Estadual de Campinas, CP 6121, \\ 13083-862 Campinas - SP, Brasil
}

Recebido em 25/8/07; aceito em 7/3/08; publicado na web em 26/8/08

\begin{abstract}
DEVELOPMENT OF AN ANALYTICAL METHOD FOR CHOLESTEROL DETERMINATION IN FEED FOR RUMINANTS USING FACTORIAL EXPERIMENTAL DESIGN. A chromatographic method was developed for cholesterol determination in feed for ruminants using response surface methodology. Among the five approaches of sample preparation methods tested, the saponification of the sample without heating presented less interference in the gas chromatography. The method presented a relative standard deviation (RSD) of $4.3 \%$, recoveries between 84 and $87 \%$ and detection limit of $0.001 \mathrm{mg}$ of cholesterol per g of feed.
\end{abstract}

Keywords: GC; extraction; validation.

\section{INTRODUÇÃO}

No Brasil, a maior parte do rebanho bovino é alimentada em sistema extensivo. Quando necessária, a suplementação alimentar é realizada utilizando fontes vegetais de energia e proteína, como o milho, a soja e o trigo; além da suplementação com constituintes minerais.

Nos organismos de origens vegetal e animal, os esteróis derivamse a partir de uma mesma molécula, o acetil-CoA, e a síntese bioquímica é idêntica até a formação do esqualeno linear. Após esta etapa, em células animais ocorre a formação de uma molécula denominada lanosterol que após cerca de 20 reações, como oxidações, perdas e migrações dos grupos metila, dá origem ao colesterol; já nas células vegetais, é formado o cicloartenol que após várias reações dá origem aos fitosteróis. ${ }^{1,2}$ Desta forma, o colesterol é o principal esterol do reino animal enquanto o $\beta$-sitosterol, o campesterol e o estigmasterol são os principais esteróis do reino vegetal. Estes compostos apresentam como estrutura química um sistema de anel tetracíclico com uma ligação dupla em um dos anéis, um grupo hidroxila no $\mathrm{C} 3$, um grupo metila nas posições 10 e 13 e um radical no carbono 17. Os fitosteróis contêm um grupo metila ou etila ou uma ligação dupla na cadeia lateral formada por 9 ou 10 carbonos, enquanto o colesterol possui 8 carbonos na cadeia lateral. Esta menor diferença na cadeia lateral resulta em grandes diferenças nas suas funções biológicas.

O colesterol é precursor dos ácidos biliares, dos hormônios esteróides da glândula supra-renal do córtex, dos hormônios sexuais masculinos e femininos e da vitamina $\mathrm{D}$, sendo também essencial ao crescimento e viabilidade das células. Nos vegetais, a função dos fitosteróis não é ainda tão bem conhecida, entretanto, sabe-se que está relacionada ao controle da permeabilidade e fluidez das membranas celulares. ${ }^{1}$

Na literatura são encontradas diversas técnicas capazes de detectar o colesterol, identificando-o com precisão e exatidão em diversos substratos; entretanto, a cromatografia constitui o método de escolha quando se requer a separação deste, dos outros esteróis também presentes na amostra. Para determinar o teor de colesterol em alimentos

\footnotetext{
*e-mail: cecibandeira@yahoo.com.br
}

podem ser utilizadas a cromatografia gasosa $(\mathrm{CG})$ ou a cromatografia líquida de alta eficiência (CLAE). Em amostras contendo colesterol e fitosteróis em pequenas quantidades, a CG é preferida por ser mais sensível que a CLAE.

Geralmente a análise de colesterol por cromatografia envolve as seguintes etapas: extração dos lipídios totais com solventes orgânicos ou mistura de solventes; remoção do solvente; saponificação alcalina dos lipídios totais; extração da matéria insaponificável com solventes orgânicos; remoção do solvente; derivação, se for utilizada a CG e, separação cromatográfica. No entanto, atualmente o método de extração mais comumente utilizado é a saponificação direta da amostra seguida da extração da matéria insaponificável com solvente orgânico e subseqüente determinação do colesterol por CG ou CLAE. Van Elsvyk et al. ${ }^{3}$ e Bragagnolo e Rodriguez-Amaya ${ }^{4}$ observaram que os teores de colesterol em amostras de ovos usando o método da extração dos lipídios totais seguido de saponificação apresentaram valores menores que o método da saponificação da amostra. A etapa da saponificação é muito importante na determinação de colesterol total desde que nesta etapa os ésteres de colesterol são convertidos em colesterol livre. Quando esta etapa não é realizada, os resultados devem ser expressos em colesterol livre para que os valores de colesterol total não sejam subestimados. Algumas plantas produzem colesterol em pequena quantidade. ${ }^{1,5} \mathrm{O}$ conceito de que produtos de origem vegetal não contêm colesterol deve-se, em parte, às pequenas concentrações encontradas e também aos métodos analíticos de detecção, que não foram bem desenvolvidos até recentemente. ${ }^{5}$

Na literatura não foi encontrado nenhum método para a determinação de colesterol em ração para ruminantes, portanto, o objetivo deste trabalho foi desenvolver e validar uma metodologia analítica para determinação de colesterol por CG utilizando planejamento experimental.

\section{PARTE EXPERIMENTAL}

\section{Material e reagentes}

$1 \mathrm{~kg}$ de ração para ruminantes foi adquirido no comércio de Campinas. Esta amostra foi homogeneizada, quarteada manualmente 
e moída em multiprocessador. Os padrões de colesterol, colestanol, 19-hidroxicolesterol, 7-pregnenolona, $\beta$-sitosterol, campesterol e estigmasterol foram adquiridos da Sigma Chemical Company (St. Louis, EUA). Todos os solventes utilizados para o preparo das amostras foram de grau analítico e para a análise por CG, de grau cromatográfico, filtrados em filtro orgânico de 0,45 $\mu \mathrm{m}$ (Millipore, Cork, Irlanda). Os gases utilizados na CG foram de alta pureza.

\section{Planejamento experimental para otimização da extração da matéria insaponificável}

Para a otimização da extração da matéria insaponificável em amostras de ração destinadas à alimentação de ruminantes foram testados inicialmente cinco métodos: saponificação direta da amostra com aquecimento; ${ }^{6}$ extração de lipídios ${ }^{7}$ seguida de saponificação com aquecimento ${ }^{8}$ hidrólise ácida ${ }^{9} \mathrm{e}$ extração dos lipídios com éter etílico utilizando equipamento de Soxhlet, seguida de saponificação com aquecimento; ${ }^{6}$ hidrólise enzimática ${ }^{10} \mathrm{e}$ extração dos lipídios com hexano, seguida de saponificação com aquecimento ${ }^{6} \mathrm{e}$, saponificação direta da amostra sem aquecimento. ${ }^{11,12} \mathrm{O}$ método que apresentou os melhores resultados considerando a área, resolução e separação do pico de colesterol, facilidade e rapidez na extração da matéria insaponificável e utilização de menor quantidade de solvente foi selecionado para a otimização através de um planejamento experimental, tendo como base a metodologia desenvolvida por Mariutti et al. ${ }^{11}$ para extração de matéria insaponificável em carne de frango.

Um planejamento experimental completo $2^{3}$, com 4 pontos centrais, totalizando 12 ensaios foi delineado e executado. Este procedimento envolveu três passos principais: a realização dos experimentos delineados estatisticamente, a estimativa dos coeficientes em um modelo matemático preditivo e a checagem da adequacidade do modelo. ${ }^{13}$ Os ensaios foram realizados em ordem aleatória. A Figura 1 apresenta um fluxograma simplificado do método de extração de matéria insaponificável, indicando as variáveis estudadas no planejamento experimental.

$$
\begin{gathered}
1 \mathrm{~g} \text { de amostra }+3 \mathrm{~g} \mathrm{KOH}+20 \mathrm{~mL} \text { de etanol } \\
\Downarrow \\
\mathrm{X}_{1} \text { horas, no escuro, } 118 \mathrm{rpm} \text {, atmosfera de } \mathrm{N}_{2} \\
\Downarrow \\
\text { Adicionar } 12 \mathrm{~mL} \text { de água destilada } \\
\Downarrow \\
\mathrm{X}_{2} \text { extrações da matéria insaponificável com } \\
10 \mathrm{~mL} \text { hexano } \\
\Downarrow \\
\text { Lavar com } \mathrm{X}_{3} \mathrm{~L} \text { de água destilada } \\
\Downarrow \\
\text { Filtrar a matéria insaponificável em sulfato } \\
\text { de sódio anidro } \\
\Downarrow \\
\text { Lavar o filtro e o papel filtro com } 10 \mathrm{~mL} \text { de hexano } \\
\Downarrow \\
\text { Secar sob } \mathrm{N}_{2}
\end{gathered}
$$

Figura 1. Fluxograma para extração de matéria insaponificável em ração para ruminantes

\section{Cromatografia gasosa}

Os extratos de matéria insaponificável foram redissolvidos em $1 \mathrm{~mL}$ de hexano e $1 \mu \mathrm{L}$ foi injetado em um cromatógrafo gasoso (Shimadzu GC-2010) com detector por ionização em chamas (DIC) e software GC Solution. Foram testadas duas colunas capilares sendo uma de polaridade intermediária com fase estacionária composta de
$14 \%$ cianopropilfenil e $86 \%$ dimetilpolissiloxano, $0,25 \mu \mathrm{m} \times 0,32 \mathrm{~mm}$ x 15 m de comprimento (DB $1701^{\circledR}$, marca J\&W) e outra coluna polar com fase estacionária de polietilenoglicol, 0,25 $\mu \mathrm{m}$ x 0,25 $\mathrm{mm}$ x $30 \mathrm{~m}$ (VA-WAX ${ }^{\circledR}$, marca Varian). Várias condições cromatográficas foram testadas, tais como tipo de injetor (split, splitless), razão do split, temperatura do injetor e do detector e programação de temperatura da coluna e fluxo. A melhor separação do pico de colesterol foi obtida com a coluna polar nas seguintes condições: injetor splitless a 260 ${ }^{\circ} \mathrm{C}$, coluna a $100{ }^{\circ} \mathrm{C}$ por $2 \min$ e a $260{ }^{\circ} \mathrm{C}$ por $48 \mathrm{~min}$, sendo que a elevação da temperatura foi de $15^{\circ} \mathrm{C}$ por min, fluxo de $1,68 \mathrm{~mL} / \mathrm{min}$ e detector a $300{ }^{\circ} \mathrm{C}$. Foram utilizados $\mathrm{H}_{2}$ (gás de arraste), $\mathrm{N}_{2}$ (make up) e ar sintético (comburente) nas seguintes proporções: 30:40:400 $\mathrm{mL} / \mathrm{min}$, respectivamente.

A identificação do colesterol foi realizada através da comparação do tempo de retenção do padrão de colesterol e por co-cromatografia. A quantificação foi realizada por padronização externa através das áreas correspondentes dos picos de padrão de colesterol desde que os padrões internos testados (colestanol, 19-hidroxicolesterol, 7-pregnenolona) co-eluiram com interferentes.

\section{Controle de qualidade analítico}

Para a validação do método foram realizados testes de especificidade, linearidade, limite de detecção, limite de quantificação, precisão e exatidão.

A linearidade foi avaliada através do coeficiente de correlação; a especificidade foi verificada através da adição de padrões (colesterol, estigmasterol, campesterol e $\beta$-sitosterol) às amostras e comparação com o tempo de retenção dos padrões. O limite de detecção foi calculado como 3 vezes o ruído da linha de base e o limite de quantificação, como 3 vezes o limite de detecção. A precisão e a exatidão foram avaliadas através do coeficiente de variação e da porcentagem de recuperação, respectivamente, ${ }^{14}$ para tanto, as amostras de ração foram adicionadas de padrão de colesterol em 6 concentrações $(0,01$; 0,$025 ; 0,05 ; 0,1 ; 0,25$ e $0,5 \mathrm{mg}$ colesterol $/ \mathrm{mL}$ ), sendo realizadas 6 repetições para cada concentração.

\section{Análise estatística}

O software Statistica for Windows 5.5 (StatSoft Inc., Tulsa, Oklahoma, USA) foi utilizado para realizar as análises de regressão, variância (ANOVA) e para obtenção das superfícies de resposta no planejamento experimental. A equação polinomial de segunda ordem expressa a seguir foi usada para correlacionar o teor de colesterol e as variáveis dependentes, sendo os dados ajustados aos resultados experimentais (nível de confiança de 95\%).

$$
y=b_{0}+\sum_{i=1}^{k} b_{i} x_{i}+\sum_{i=1}^{k} b_{i} x_{i}^{2}+\sum_{i=1}^{k-1} \sum_{j=i+1}^{k} b_{i j} x_{i} x_{j}
$$

$(\mathrm{i}=1-3, \mathrm{j}=1-3)$

As repetições dos parâmetros utilizados na validação foram avaliadas através da média, desvio padrão e coeficiente de variação obtidos com o programa Microsoft Excel.

\section{RESULTADOS E DISCUSSÃO}

\section{Condições cromatográficas}

A maioria dos trabalhos encontrados na literatura para determinação de colesterol por CG em alimentos utiliza coluna com fase estacionária apolar (5\% difenil e 95\% dimetilpolissiloxano). Neste 
trabalho foi utilizado inicialmente uma coluna de polaridade intermediária (DB $1701^{\oplus}, \mathrm{J} \& \mathrm{~W}$ ) com fase estacionária composta de $14 \%$ cianopropilfenil e $86 \%$ dimetilpolissiloxano e $15 \mathrm{~m}$ de comprimento. $\mathrm{Na}$ tentativa de quantificar o colesterol através do método da padronização interna, usando colestanol como padrão interno, com este tipo de coluna não houve separação dos picos, mesmo alterando-se parâmetros tais como razão do split, fluxo, temperatura do detector e diferentes programações de temperatura da coluna. Utilizou-se uma coluna polar VA-WAX ${ }^{\circledR}\left(30 \mathrm{~m}\right.$ x $0,25 \mathrm{~mm}^{\circ} 0,25 \mu \mathrm{m}$ de polietilenoglicol, Varian) obtendo-se boa separação entre o colesterol e o colestanol. No entanto quando foi analisada a ração, o colestanol eluiu junto com um interferente da amostra, inviabilizando a quantificação com este padrão interno. Foram testados mais dois padrões, 19-hidroxicolesterol e 7-pregnenolona, os quais também eluíram junto com interferentes da amostra. Desta forma, a quantificação foi realizada por padronização externa.

Ahmida et al. ${ }^{15}$ relataram este mesmo problema na separação entre colesterol e colestanol na determinação de fitosteróis em plasma sanguíneo usando uma coluna apolar, por outro lado, conseguiram boa separação dos fitosteróis de interesse utilizando uma coluna de polaridade intermediária (14\% cianopropilfenil e $86 \%$ dimetilpolissiloxano), semelhante à utilizada nos testes iniciais deste experimento, porém, mais longa $(60 \mathrm{~m})$.

Dutta e Normém ${ }^{16} \mathrm{e}$ Phillips et al. ${ }^{17}$ também utilizaram coluna de polaridade intermediária (14\% cianopropilfenil e $86 \%$ dimetilpolissiloxano) para determinação de esteróis. Os primeiros autores realizaram a determinação de fitosteróis saturados em aveia e os segundos, de fitosteróis, estanóis e metabólitos do colesterol em soro sanguíneo e obtiveram excelente separação dos compostos, entretanto, nestes dois trabalhos não foi realizada a determinação de colesterol. Poucos trabalhos foram encontrados na literatura utilizando colunas polares para determinação de esteróis, e nenhum deles utilizou coluna polar de polietilenoglicol para determinação de colesterol. Frega et al. ${ }^{18}$ compararam a separação de fitosteróis em azeite de oliva utilizando coluna polar e apolar e constataram que a primeira foi mais eficiente porque melhorou a resolução dos picos de esteróis, além de apresentar picos não detectados com a coluna apolar. Rodriguez-Estrada et al. ${ }^{19}$ também compararam a utilização de coluna polar e apolar e observaram uma melhor separação da matéria insaponificável de extratos lipídicos com a coluna polar. Por outro lado, Lagarda et al..$^{20}$ compararam diversas colunas cromatográficas e concluíram que para a análise de colesterol é preferível utilizar coluna apolar composta de $5 \%$ difenil e $95 \%$ dimetilpolissiloxano (60 m x $0.1 \mathrm{~m} \mathrm{x} 0.25 \mathrm{~mm}$ ), enquanto que para outros esteróis, outras fases estacionárias, de polaridade intermediária, também são apropriadas, como, por exemplo, a de $14 \%$ cianopropilfenil e $86 \%$ dimetilpolissiloxano. Entretanto, os autores não relataram qual a melhor coluna para separar, em uma mesma amostra, o colesterol dos outros esteróis.

\section{Otimização da extração da matéria insaponificável para determinação de colesterol}

Atualmente, o método mais utilizado para a determinação de colesterol em alimentos consiste da saponificação com aquecimento da amostra seguida da extração da matéria insaponificável com solventes orgânicos e quantificação por cromatografia gasosa (CG) ou cromatografia líquida de alta eficiência (CLAE). Considerando que ração para ruminantes é uma matriz extremamente complexa, foram testados cinco métodos de preparo de amostra. Com o objetivo de obter um extrato de colesterol com o menor número de interferentes possível testou-se o método da extração de lipídios a frio ${ }^{7}$ e saponificação dos lipídios com aquecimento ${ }^{7}$ o qual se mostrou insatisfatório, uma vez que a área do pico do colesterol nas amostras de ração foi muito pequena e, além disso, consumiu grande quantidade de solventes, apresentou várias etapas em sua execução, sendo, portanto, muito laborioso e demorado. Em adição, utilizou como solventes o clorofórmio e o metanol, os quais hoje em dia são evitados para preservação do meio ambiente e da saúde humana.

Com o intuito de obter a extração total do colesterol da matriz, o menor número de interferentes, bem como solubilização total das amostras de ração, foram testados os métodos de hidrólise ácida e de hidrólise enzimática. A hidrólise ácida seguida da extração dos lipídios por Soxhlet e saponificação dos lipídios com aquecimento apresentou um cromatograma com o pico de colesterol eluindo em tempo de retenção muito próximo ao dos picos interferentes, além de ser um método bastante laborioso por apresentar muitas etapas em sua execução. A hidrólise enzimática promoveu aumento na área do colesterol em relação aos métodos testados anteriormente, no entanto, aumentou também a quantidade de interferentes próximos ao pico do colesterol e não houve solubilização total da amostra. Este aumento da concentração de colesterol nas amostras obtidas por hidrólise enzimática pode ter ocorrido em função da liberação de óleo da parede celular de células vegetais. Ferrari e Santos ${ }^{21}$ propuseram um método de hidrólise enzimática para extração de lipídios totais da soja, utilizando diversas enzimas, uma vez que o óleo da parede celular muitas vezes não é adequadamente extraído com solventes. Comparando a saponificação direta da amostra com e sem aquecimento, no método da saponificação com aquecimento, apesar de serem utilizadas diferentes quantidades de amostras e reagentes, após a aplicação de calor, houve a formação de uma solução viscosa e densa nas amostras de ração, inviabilizando sua continuidade. Além disso, não houve solubilização total das amostras de ração. O método da saponificação direta da amostra sem aquecimento foi o que apresentou um cromatograma com menor número de interferentes e maior área do pico de colesterol, além de ser de fácil execução e utilizar menor quantidade de solvente. Este método foi, portanto, selecionado para a otimização através de planejamento experimental.

A escolha das variáveis independentes tempo de saponificação, número de extrações da matéria insaponificável e volume de água necessário para neutralizar o extrato hexânico (Tabela 1) para o delineamento do planejamento fatorial completo $2^{3}$ foi baseada no trabalho de Mariutti et al. ${ }^{11}$ e em testes preliminares. Nos testes preliminares foi constatado que tempos inferiores a $23 \mathrm{~h}$ não eram suficientes para a completa dissolução e saponificação da amostra, portanto, $23 \mathrm{~h}$ foi definido como nível mínimo $(-1)$, e os demais níveis $(0$ e +1$)$ com um intervalo de $2 \mathrm{~h}$ entre si. O número ideal de extrações obtido por Mariutti et al. ${ }^{11}$ para carne de frango foi de 4, assim, este mesmo número de extrações foi estabelecido como nível máximo (+1) uma vez que a quantidade estimada de colesterol presente na ração é menor. Quanto à completa neutralização do extrato hexânico, verificou-se esta necessidade dado que os extratos com $\mathrm{pH} 9$ resultavam em cromatogramas com maior quantidade de interferentes.

Os coeficientes de regressão para o modelo codificado são apresentados na Tabela 2. De acordo com a análise de variância realizada

Tabela 1. Variáveis independentes do planejamento fatorial completo $2^{3}$

\begin{tabular}{lccc}
\hline Variáveis independentes & \multicolumn{3}{c}{ Níveis } \\
& -1 & 0 & +1 \\
\hline $\mathrm{X}_{1}$ - Tempo de saponificação $(\mathrm{h})$ & 23 & 25 & 27 \\
$\mathrm{X}_{2}$ - Número de extrações & 2 & 3 & 4 \\
$\mathrm{X}_{3}$ - Água para retirada do KOH $(\mathrm{L})$ & 1 & 1,5 & 2 \\
\hline
\end{tabular}


Tabela 2. Coeficientes de regressão do modelo codificado

\begin{tabular}{lcc}
\hline Fator & $\begin{array}{c}\text { Coeficiente de } \\
\text { Regressão }\end{array}$ & $\mathrm{p}$ \\
\hline Média & 257080 & $1,9 \times 10^{-6}$ \\
Número de extrações (n) & 22865 & 0,66 \\
Tempo de saponificação $(\boldsymbol{h})$ & -12305 & 0,03 \\
Vol. água retirada do $\mathbf{K O H}(\boldsymbol{l})$ & -1437 & $4,6 \times 10^{-3}$ \\
$\boldsymbol{n} \boldsymbol{x} \boldsymbol{h}$ & 8380 & 0,01 \\
$\boldsymbol{n} \boldsymbol{x} \boldsymbol{l}$ & 10028 & 0,04 \\
$\mathrm{~h} \times \mathbf{l}$ & -16027 & 0,07 \\
\hline
\end{tabular}

As variáveis e interações em negrito e itálico são estatisticamente significativas $(\mathrm{p}<0,05)$.

para os resultados obtidos foi verificado que o modelo é preditivo $\left(\mathrm{r}^{2}=67 \%, \mathrm{p}<0,05\right)$.

$\mathrm{O}$ número de extrações não foi estatisticamente significativo ( $>0,05)$, porém a interação desta variável com o tempo de saponificação e o volume de água para neutralização do extrato hexânico apresentaram efeitos positivos e estatisticamente significativos. A análise das interações entre estas variáveis indica que um maior número de extrações, em conjunto com um maior volume de água para neutralização do extrato hexânico e menor tempo de saponificação serão obtidos os maiores teores de colesterol. Por outro lado, os efeitos negativos apresentados pelas variáveis tempo de saponificação e volume de água para neutralização do extrato hexânico indicam que o aumento no tempo de saponificação e no volume de água para neutralização, quando analisados isoladamente, causam diminuição no teor de colesterol.

As condições otimizadas para a determinação de colesterol em ração para ruminantes foram definidas da seguinte forma: pesar 1,0 $\mathrm{g}$ de amostra e saponificar com $3 \mathrm{~g}$ hidróxido de potássio em pastilhas e $20 \mathrm{~mL}$ de álcool etílico durante $23 \mathrm{~h}$, na ausência de luz, a temperatura ambiente, sob agitação a $118 \mathrm{rpm}$ em atmosfera de $\mathrm{N}_{2}$. Após a saponificação, adicionar $12 \mathrm{~mL}$ de água destilada e realizar 4 extrações da matéria insaponificável com $10 \mathrm{~mL}$ de hexano cada. A seguir, lavar o extrato hexânico com $2 \mathrm{~L}$ de água destilada, para retirada do $\mathrm{KOH}$, filtrar com $3 \mathrm{~g}$ sulfato de sódio anidro e lavar o filtro com $10 \mathrm{~mL}$ de hexano. Secar o extrato hexânico contendo a matéria insaponificável sob fluxo de nitrogênio. Redissolver o resíduo em $1 \mathrm{~mL}$ de hexano, filtrar e injetar no CG nas condições definidas anteriormente.

O colesterol exibiu linearidade na faixa de concentração de 0,01 a $0,5 \mathrm{mg} / \mathrm{mL}$ com coeficientes de determinação $\left(\mathrm{r}^{2}\right)$ de 0,9999 . A especificidade do método foi verificada através da separação cromatográfica dos padrões de colesterol, $\beta$-sitosterol, campesterol e estigmasterol (Figura 2). O coeficiente de variação entre 4 amostras analisadas no mesmo dia nas condições otimizadas foi de 4,3\%, demonstrando boa repetibilidade do método. O limite de detecção e o limite de quantificação para o colesterol foram de 0,001 e 0,003 $\mathrm{mg} / \mathrm{g}$, respectivamente. A recuperação e o coeficiente de variação variaram de 84 a $87 \%$ e de 2,9 a 4,0\%, respectivamente, em relação às diferentes concentrações de colesterol $(0,01 ; 0,025 ; 0,05 ; 0,1 ; 0,25$ e $0,5 \mathrm{mg}$ colesterol $/ \mathrm{mL}$ ) adicionadas nas rações.

O método foi aplicado para a determinação de colesterol em 10 amostras de diferentes procedências obtendo-se valores entre 0,0110 a $0,0222 \mathrm{mg} / \mathrm{g}$ com valor médio de $0,0176 \pm 0,0042$, muito superior ao limite de quantificação do método proposto. Algumas plantas produzem colesterol em pequena quantidade; ${ }^{1,5}$ considerando que os ingredientes normalmente utilizados nas rações animais são o milho, o farelo de soja e o farelo de trigo, o valor obtido está coerente com dados encontrados na literatura. ${ }^{5,17,22}$

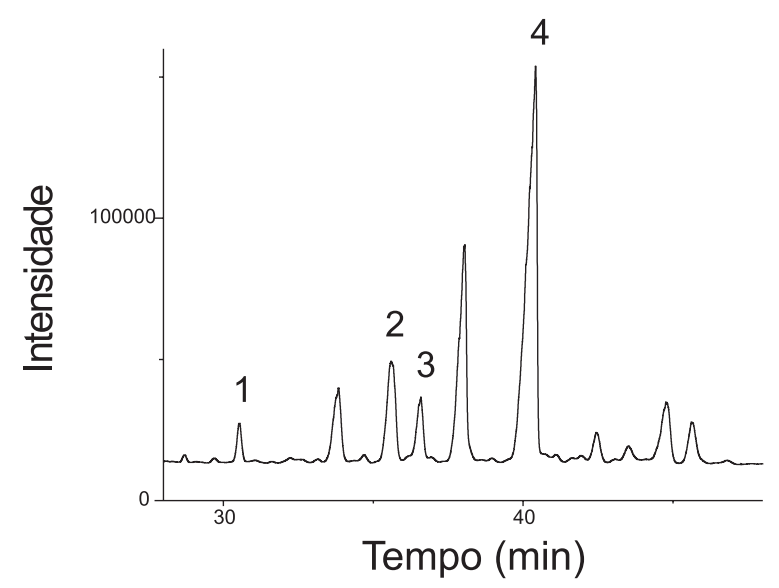

Figura 2. Cromatograma de amostra de ração, obtido por CG-DIC, coluna VA $W A X^{\circledR}$, no tempo de 25 a 50 min. Condições cromatográficas: no texto. Picos: (1) colesterol; (2) campesterol; (3) estigmasterol; (4) $\beta$-sitosterol

\section{CONCLUSÃO}

O método desenvolvido para determinação de colesterol em ração animal mostrou-se exato e preciso, sendo de fácil execução e utilizando pequenas quantidades de solventes.

\section{MATERIAL SUPLEMENTAR}

São apresentadas as superfícies de resposta para o planejamento fatorial completo $2^{3}$, disponível gratuitamente em http://quimicanova. sbq.org.br, na forma de arquivo PDF.

\section{AGRADECIMENTOS}

À FAPESP, CNPq e CAPES pelo suporte e apoio financeiro.

\section{REFERÊNCIAS}

1. Piironen, V.; Lindsay, D. G.; Miettinen, T. A.; Toivo, J.; Lampi, A. M.; J. Sci. Food Agric. 2000, 80, 939.

2. Volin, P.; J. Chromatogr., A 2001, 935, 125.

3. Van Elswyk, M. E.; Schake, L. S.; Hargis, P. S.; Poultry Sci. 1991, 70, 1258.

4. Bragagnolo, N.; Rodriguez-Amaya, D. B.; J. Food Comp. Anal. 2003 $16,147$.

5. Behrman, E. J.; Gopalan, V.; J. Chem. Educ. 2005, 82, 1791.

6. Saldanha, T.; Mazalli, M. R.; Bragagnolo, N.; Ciênc.Tecnol. Aliment. 2004, 24, 109.

7. Folch, J.; Less, M.; Stanley, S.; J. Biol. Chem. 1957, 226, 497.

8. Bohac, C. E.; Rhee, K. S.; Cross, H. R.; Ono, K.; J. Food Sci. 1988, 5 , 1642.

9. Diemair, W.; Laboratoriumsbuch fur Lebensmittelchemiker, $8^{\text {th }}$ ed., Verlag Von Theodor Steinkopff: Dresden, 1963.

10. Rios, A. O.; Mercadante, A. Z.; Food Addit. Contam. 2004, 21,125.

11. Mariutti, L. R. B.; Nogueira, G. C.; Bragagnolo, N.; J. Agric. Food Chem., no prelo.

12. Mazalli, M. R.; Sawaya, A. C. H. F.; Eberlin, M. N.; Bragagnolo, N.; Lipids 2006, 41, 615.

13. Box, G. E. P.; Hunter, W. G.; Hunter, J. S.; Statistics for Experiments: An Introduction to Designs, Data Analysis and Model Building, Wiley: New York, 1978. 
14. Comissão da União Européia; Directiva 2002/657/CE de 12 de agosto de 2002; J. Off. Comun. Europ. 2002, L 221, 8.

15. Ahmida, H. S. M.; Bertucci, P.; Franzo, L.; Massoud, R.; Cortese, C.; Lala, A.; Federici, G.; J. Chromatogr., B: Anal. Technol. Biomed. Life Sci. 2006, 842, 43.

16. Dutta, P. C.; Normém, L.; J. Chromatogr., A 1998, 816, 177; Phillips, K. M.; Ruggio, D. M.; Bailey, J. A.; J. Chromatogr., B: Anal. Technol. Biomed. Life Sci. 1999, 732, 17.

17. Phillips, K. M.; Ruggio, D. M.; Toivo, J. I.; Swank, M. A.; Simpkins, A. H.; J. Food Comp. Anal. 2002, 15, 123.
18. Frega, N.; Bocci, F.; Lercker, G.; J. Am. Oil Chem. Soc. 1992, 69, 447.

19. Rodriguez-Estrada, M. T.; Frega, N.; Lercker, G.; Grasas Aceites 2002 , $53,76$.

20. Lagarda, M. J.; García-Llatas, G.; Farré, R.; J. Pharm. Biomed. Anal. 2006, 41, 1486.

21. Ferrari, A. M.; Santos, J.; Ciên. Tecnol. Aliment. 2005, 25, 132.

22. Rossell, J. B.; Analysis of oilseeds, fats and fatty foods, Elsevier: London, 1991. 


\section{DESENVOLVIMENTO DE METODOLOGIA ANALÍTICA PARA DETERMINAÇÃO DE COLESTEROL EM RAÇÃO PARA RUMINANTES ATRAVÉS DE PLANEJAMENTO EXPERIMENTAL FATORIAL}

Cecília Muller Bandeira* e José Maria Ferreira

Departamento de Tecnologia e Inspeção de Produtos de Origem Animal, Escola de Veterinária, Universidade Federal de Minas Gerais, Av. Antônio Carlos, 6627, 31270-901 Belo Horizonte - MG, Brasil

Neura Bragagnolo e Lilian Regina Barros Mariutti

Departamento de Ciência de Alimentos, Faculdade de Engenharia de Alimentos, Universidade Estadual de Campinas, CP 6121, 13083-862 Campinas - SP, Brasil
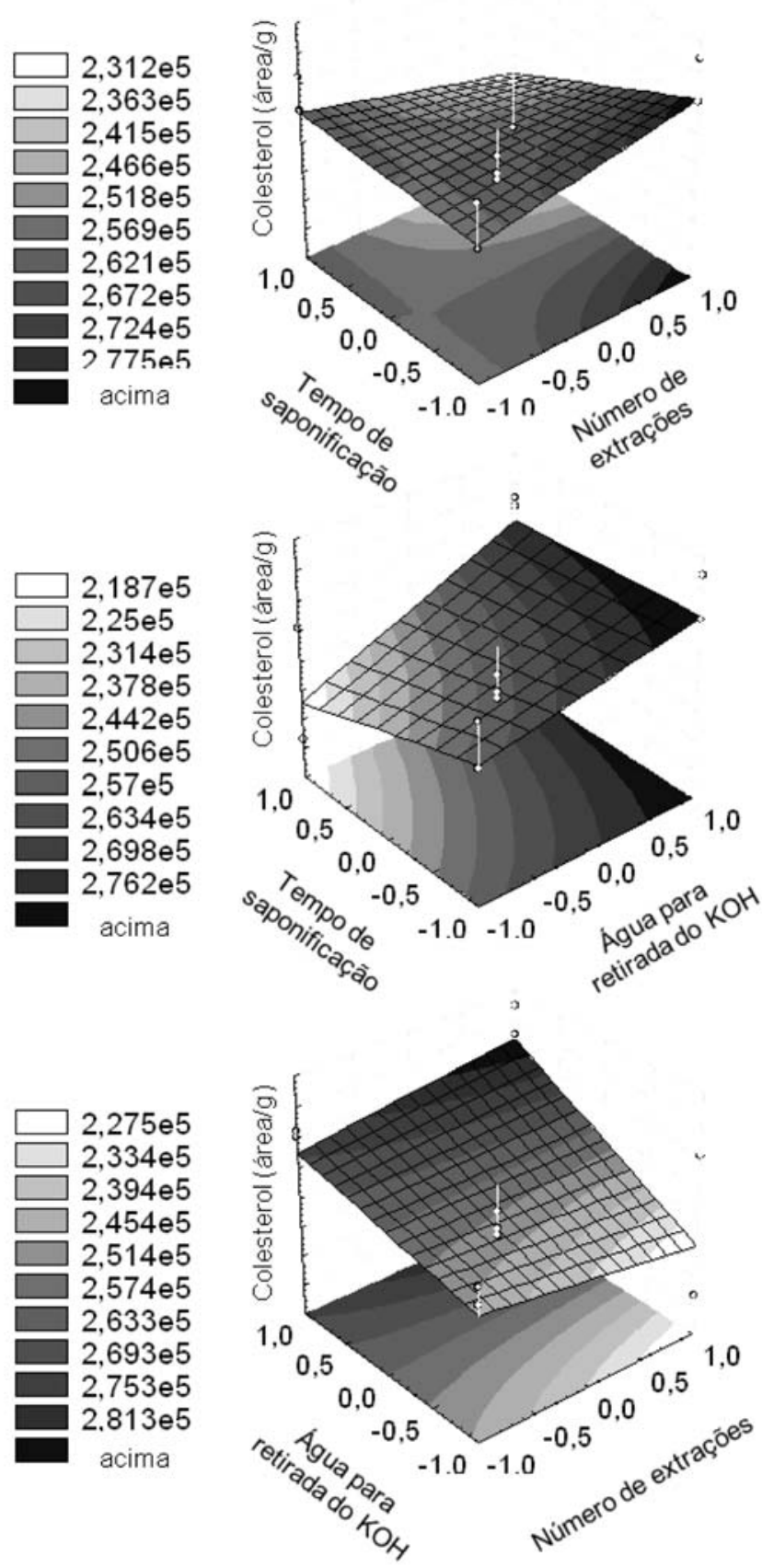

Figura 1S. Superfícies de resposta para o planejamento fatorial completo $2^{3}$

*e-mail: cecibandeira@yahoo.com.br 